\title{
The Effect of Smoking on the Physical Fitness of Elderly Male Subjects
}

\author{
Hüseyin Eroğlu ${ }^{1, *}$, Selami Yüksek² \\ ${ }^{1}$ High School of Physical Education and Sport, Kahramanmaraş Sütçü İmam University, Turkey \\ ${ }^{2}$ High School of Physical Education and Sport, Karadeniz Teknik University, Turkey
}

Copyright $\odot 2018$ by authors, all rights reserved. Authors agree that this article remains permanently open access under the terms of the Creative Commons Attribution License 4.0 International License

\begin{abstract}
Introduction: This study was conducted to analyze the effect of the smoking habit on the physical fitness of elderly male subjects. Material and Method: The study was conducted on 849 (age $68.7 \pm 6.1$ years, height $169.4 \pm 6.4 \mathrm{~cm}, 76.4 \pm 11.00 \mathrm{~kg}$ ) elderly male volunteers who could independently perform their daily activities and did not suffer from any health problems. These subjects were divided into three groups as smokers $(n=239)$, smoking quitters $(n=285)$ and non-smokers $(n=325)$. In this study, "Senior Fitness Test" (SFT) protocols comprising of six tests were used to determine the lower and upper extremity strength, flexibility, dynamic balance, coordination and aerobic endurance. The data analysis was performed based on the age groups of participants, and the significance of differences between groups, i.e. "smokers (S)-non-smokers (NS)", "smokers (S)-smoking quitters (SQ)" and "non-smokers (NS)-smoking quitters (SQ)", were determined using "Independent Samples T" test. Findings: The data analysis suggests that non-smokers of all ages had higher physical fitness performance scores compared to smokers, which was a statistically significant finding $(p<0.05)$. Conclusion: It was found out that non-smoking elderly male subjects had higher physical fitness level compared to smoking elderly male subjects in all tests.
\end{abstract}

\section{Keywords Physical Fitness, Smoking, Ageing}

\section{Introduction and Objective}

It is important that the physical fitness, which affects the efficacy in daily life and sportive activities, is determined in early ages [1]. Both physical activities and exercise bear a healing and supporting effect for diseases as well as preventing them [2]. They mainly aim to offer a healthy lifestyle and keep an individual's physical condition at a suitable level [2]. Exercise plays an important role in the functional capacity due to the physiological changes that it brings about. Flexibility and strength training prevent muscle weakness as well as walking and balance disorders [2]. Balance and walking disorders caused by muscle weakness are primary risk factors for falls in elderly people [3].

It is widely known that the changes in physical fitness of elderly people due to ageing occur differently for physically active and inactive people. It has been reported that exercise significantly increases life quality and physical fitness levels of elderly people and that it bears a preventive and healing effect on various diseases as well as extending average lifespan $[2,4,5]$. Even though physical differences that occur due to ageing can be observed, it is still difficult to distinguish between the physical differences (e.g. height, body weight, strength, and flexibility) of two individuals aged 20 and 70 sitting on a chair. Physiological (e.g. heart rate, blood pressure) differences can be observed when an individual moves, reacts to a sound alarm or needs to change his/her position as quickly as possible [6].

Today's societies suffer from a number of health problems such as coronary heart disease, obesity, stress, hypertension, diabetics, osteoporosis, cancer and emotional disorders. The emergence of health problems can be also associated with modern lifestyles with a low level of physical activities. It was reported that increasing levels of physical activity increased life quality for elderly people and delayed the physical inabilities. Even chronic patients improve their physical functions through regular participation in physical activities $[6,7]$.

Exercise and physical activity are also related to smoking habit [8]. However, the number of studies focusing on the quantitative correlation is relatively low in the relevant literature [8]. The percentage of male smokers in developed countries such as Japan is quite high, which is 53.5 percent [8]. In addition, the number of current smokers tends to decrease in parallel with ageing. A study demonstrates that smokers who do regular exercise are more likely to quit smoking compared to those who do not do regular exercise [8]. As a result, it can be inferred that regular exercise influences smoking habits [8]. For 
example, when an individual becomes short of breath during regular exercise before quitting smoking, the observed phenomenon is reasonable [8]. Hierarchical regression models indicated that smoking was a predictor of the baseline 6-min walk as smokers walked significantly shorter distances compared to non-smokers [9]. In addition, smoking was found to be a significant predictor of addiction; however, the overall model that included smoking as a predictor did not display a significant effect on addiction [9]. Modifiable risk factors such as physical inactivity and smoking may endanger women in serious infections although the causal link is yet to be explained [9]. Further research in this field may propose new strategies that reduce the serious burden of infections on the elderly population [10].

This study was carried out to analyze the impact of the smoking habit on the physical fitness of elderly male subjects.

\section{Material and Method}

\section{Participants}

This study was carried out with the voluntary participation of 849 (age $68.7 \pm 6.1$ years, height $169.4 \pm 6.4$ $\mathrm{cm}, 76.4 \pm 11.00 \mathrm{~kg}$ ) men aged 60 years or more who were able to independently conduct their normal daily activities and who did not have any serious health problems. All participants provided written consent in accordance with the declaration of 1964 Helsinki, after reading the verbal and written explanations of the potential risks of the study. The participants were informed about the procedure of the experiment and were told that at any given time they could voluntarily withdraw from the experiment. Certain demographic information was obtained about the participants via a questionnaire, including whether they smoking status. Participants in the study were divided into 3 groups as smokers, quit smoking, and non-smokers. Subjects who had orthopedic or mental disabilities or neurologic or advanced cardiovascular chronic illnesses that might impair or limit test protocols were not included in the study. In performing the physical fitness tests, any clothing that could impede or limit movements, or items like jackets, coatsor, toppers were removed. In order for each subject to be proceeded through the fitness stations efficiently, an experienced assistant was employed for each station. Any subjects who were unable to complete any of the stations included in the test protocol were excluded from the evaluation.

Distribution of the participants after eliminating those not qualified. The distribution of the 849 participants who were found to be suitable for the test criteria and successfully completed the tests are as follows, according to their age range and whether they performed smoking or not: age 60- 64 (69 smokers, 74 non-smokers and 60 smoking quitters); age $65-69$ (87 smokers, 93 non-smokers and 98 smoking quitters); age $70-74$ (45 smokers, 90 non-smokers and 75 smoking quitters ); and age over 75 (38 smokers, 68 non-smokers and 52 smoking quitters).

\section{Measurements}

The body mass index (BMI) of the subjects was measured by dividing their weight into the square of their height in meters. Their pulse counts were measured by palpating the artery from the wrist for 15 seconds and multiplying it by 4 , and then recorded as pulse/minute. Their effort pulse counts were also measured using the same immediately after completion of the two-minute step test (2 MST) and recorded in the same fashion.

\section{Applied Tests}

The six-station Senior Fitness Test (SFT) developed by Rikli and Jones (2001) was applied in order to determine the physical fitness levels of participants. The subjects were put through a 4-5 minute warm-up period (walking, jog-trot, and stretching exercises) before the testing protocol was carried out. The stations constituting test protocol included:

1. Chair Stand Test (30CST): This test was performed in order to determine the lower extremity strengths of subjects. The subject sat upright, feet on the floor and arms crossed over his chest (right hand on left shoulder, left hand on right shoulder) on the centre of a $43.18 \mathrm{~cm}$ chair. The test was demonstrated and explained to the subject. After having him perform some trials to make sure that he understood the test correctly, a start command was given, and the subject performed compete rising as often as he could in 30 seconds. The number of complete risings the subject could manage in 30 seconds was recorded as his 30CST score (8).

2. Arm Curl Test (ARCLT): This test was applied in order to determine upper extremity strength. The subject sat on his dominant arm side of the chair. The subject completed the test by lifting the $3.63 \mathrm{~kg}$ dumbbell to complete extension to the floor and to complete flexion upward with his dominant hand for 30 seconds. The number of full lifts he managed in 30 seconds was recorded as his ARCLT score (8).

3. Two Minute Step Test (2MST): This test was applied in order to determine the aerobic endurance of the subjects. While the subject was standing straight, the distance between his front iliac crest and midpoint of his patella was measured. The distance between the midpoint of this distance and the floor was measured, and his step height was determined. In order to determine whether his step 
height could reach the desired level (the height of his knees), a tape was set up at the subject's knee level. The subject was asked to step barefoot on the same spot and reach the determined height on a 1.5 $x 1.5$ mat for 2 minutes. The subject was asked to start the test with his right foot, making his steps as fast as possible without running. If any step (right or left) did not reach the determined height, those steps were declared null. At the end of two minutes, the number of right foot steps that reached the determined height was recorded as the 2MST score of the participant (8).

4. 8 Foot Up-and-Go Test (8FUAGT): This test was applied in order to determine the dynamic balance and agility of the subjects. The subject sat leaning back, feet on the floor and both arms on his lap, in the centre of a $43.18 \mathrm{~cm}$ chair. The subject was asked to get up from the chair, turn around the funnel that was placed at a $2.44 \mathrm{~m}$ distance and return to sit on the chair as quickly as he could. The subject was prompted with a start command, and when he had turned the funnel and sat back down on the chair, the timer was stopped and the time he took to perform the task was recorded in seconds. Each subject performed the test twice, and the best trial was recorded as the test score (8).

5. Chair Sit and Reach Test (CSRT): This test was applied to determine the flexibility of the hamstring muscle group. The subject sat on the point where his thighbone and haunch bone met on the edge of the $43.18 \mathrm{~cm}$ chair, with one of his feet (right or left) flexed at 90 degrees and his sole pressing the floor; his other foot at full extension and his heel pressing the floor. The subject was asked to lean forward toward his toes with his hands, both hands at pronation, one on the top of other and his middle fingers in a line, not bending his knee. If the subject's fingertips did not touch his toes, the distance between them was recorded (-); if they did touch (0), and if they passed his toes $(+)$, the distance was recorded in centimeters. Both legs of the subject were tested, and the best evaluation was recorded as the test score (8).

6. Back Scratch Test (BST): This test was applied in order to determine the upper extremity flexibility, and in particular the shoulder flexibility of the subjects. Each subject stood in an anatomic standing position and was asked to reach the lowest point on his back with one of his arms over his shoulder, his palm facing his back and his fingers at extension. With his other arm, he was asked to reach the highest point on his back, with his palm facing the opposite way and his fingers at extension, and place the fingers of both hands as close together as possible. The distance between his fingertips was recorded. If the middle fingers did not touch, the distance was recorded as (-) centimeters, if the middle fingers touched end to end, the distance was recorded as zero (0), and if one of the middle fingers passed the surface of the other middle finger, the distance was recorded as $(+)$ centimeters. Both of his arms (right arm upper, left arm lower and right arm lower, left arm upper) were measured with this method, and his best evaluation was recorded as the score of this test (8).

\section{Statistical Analysis}

'One-Simple Kolmogorov Smirnov' test was used to ensure the normal distribution of the data, and it was observed that all data were normally distributed. Based on the age groups of the subjects, the significance of differences between their age, height, weight, BMI, resting and effort heart rates and test performances were analyzed "Independent Samples t". The statistical significance for all statistical analyses was taken as 0.05 , and these analyses were performed via SPSS 22.0 package program.

\section{Findings}

Table 1. Some Descriptive Facts of the Surveys

\begin{tabular}{|c|c|c|}
\hline Education status & $\mathbf{N}$ & $\%$ \\
\hline Non-literate & 133 & $\% 15.7$ \\
\hline Literate & 109 & $\% 12.8$ \\
\hline Primary School & 421 & $\% 49.6$ \\
\hline Middle School & 70 & $\% 8.2$ \\
\hline High School & 69 & $\% 8.1$ \\
\hline University & 47 & $\% 5.5$ \\
\hline \multicolumn{3}{|c|}{ Where do you live? } \\
\hline Big City & 381 & $\% 44.9$ \\
\hline City & 161 & $\% 19.0$ \\
\hline Town & 179 & $\% 21.1$ \\
\hline Village & 128 & $\% 15.1$ \\
\hline \multicolumn{3}{|c|}{ Do you smoke? } \\
\hline Yes & 239 & $\% 28,2$ \\
\hline No & 325 & $\% 38,3$ \\
\hline Quit & 285 & $\% 33,6$ \\
\hline \multicolumn{3}{|c|}{ How many years have you been smoking? } \\
\hline $7-10$ years & 1 & $\% 0,4$ \\
\hline Over 10 year & 238 & $\% 99,6$ \\
\hline \multicolumn{3}{|c|}{ How much do you smoke per day? } \\
\hline $5-10$ piece & 61 & $\% 25,8$ \\
\hline 1 package & 136 & $\% 56,9$ \\
\hline Over 1 package & 42 & $\% 17,6$ \\
\hline \multicolumn{3}{|c|}{ How many years have you smoking quitters? } \\
\hline Less than 1 year & 21 & $\% 7,4$ \\
\hline $1-3$ year & 26 & $\% 9,1$ \\
\hline 4-6 year & 50 & $\% 17,5$ \\
\hline $7-10$ year & 87 & $\% 30,5$ \\
\hline Over 10 year & 101 & $\% 35,4$ \\
\hline
\end{tabular}

Work volunteer participation of 849 healthy elderly male subjects who can independently perform their daily living activities. Frequency distributions related to education, living, smoking, quitting and daily cigarette consumption of the cases participating in the study are given in Table 1. 
Table 2. The Comparison of Some Physical and Physiological Features of Smokers and Non-Smokers Based on Their Age Groups

\begin{tabular}{|c|c|c|c|c|c|c|c|c|c|c|c|}
\hline \multirow{2}{*}{\multicolumn{2}{|c|}{$\begin{array}{c}\text { AGE GROUPS } \\
\begin{array}{c}\text { PARAMETERS } \\
\% \mathrm{~N}\end{array}\end{array}$}} & \multicolumn{2}{|c|}{$\begin{array}{c}\text { Aged 60-64 } \\
\mathrm{N}=143\end{array}$} & \multicolumn{2}{|c|}{$\begin{array}{c}\text { Aged 65-69 } \\
\text { N }=180\end{array}$} & \multicolumn{2}{|c|}{$\begin{array}{c}\text { Aged 70-74 } \\
N=135\end{array}$} & \multicolumn{2}{|c|}{$\begin{array}{l}\text { Over } 75 \\
N=106 \\
\end{array}$} & \multicolumn{2}{|c|}{$\begin{array}{c}\text { Total } \\
\mathrm{N}=564\end{array}$} \\
\hline & & \multirow{2}{*}{$\begin{array}{c}\mathbf{S} \\
\mathrm{n}=69 \\
\% 48.3 \\
61.6 \\
(1.4)\end{array}$} & \multirow{2}{*}{$\begin{array}{c}\mathbf{N S} \\
\mathrm{n}=74 \\
\% 51.7\end{array}$} & \multirow{2}{*}{$\begin{array}{c}\mathbf{S} \\
\mathrm{n}=87 \\
\% 48,3\end{array}$} & \multirow{2}{*}{$\begin{array}{c}\begin{array}{c}\text { NS } \\
\mathrm{n}=93 \\
\% 51.7\end{array} \\
66.4 \\
(1.3) \\
\end{array}$} & \multirow{2}{*}{$\begin{array}{c}\mathbf{S} \\
\mathrm{n}=45 \\
\% \\
33.3 \\
71.6 \\
(1.3)\end{array}$} & \multirow{2}{*}{$\begin{array}{c}\begin{array}{c}\mathbf{N S} \\
\mathrm{n}=90 \\
\% 66.7\end{array} \\
71.4 \\
(1.5)\end{array}$} & \multirow{2}{*}{$\begin{array}{c}\begin{array}{c}\mathbf{S} \\
\mathrm{n}=38 \\
\%\end{array} \\
35.8 \\
77.2 \\
(2.9)\end{array}$} & \multirow{2}{*}{$\begin{array}{c}\mathbf{N S} \\
\mathrm{n}=68 \\
\% 64.2\end{array}$} & \multirow{2}{*}{$\begin{array}{c}\begin{array}{c}\mathbf{S} \\
\mathrm{n}=239 \\
\%\end{array} \\
42.4 \\
67.7 \\
(5.6)\end{array}$} & \multirow{2}{*}{$\begin{array}{c}\text { NS } \\
\mathrm{n}=325 \\
\% 57.6 \\
69.2 \\
(6.4)\end{array}$} \\
\hline \multirow{3}{*}{ Age Years } & $\begin{array}{c}\mathrm{X} \\
(\mathrm{SS})\end{array}$ & & & & & & & & & & \\
\hline & $\mathrm{AD} \pm \mathrm{SD}$ & \multirow{2}{*}{\multicolumn{2}{|c|}{$\begin{array}{c}0.1 \pm 0.2 \\
0.628\end{array}$}} & & & & & & 0.7 & -1.5 & $=0.5$ \\
\hline & $\mathrm{p}$ & & & \multicolumn{2}{|c|}{$\frac{0.1+.2}{0.722}$} & \multicolumn{2}{|c|}{$\frac{0.2 .0}{0.354}$} & \multicolumn{2}{|c|}{$0.018^{*}$} & \multicolumn{2}{|c|}{$0.004 * *$} \\
\hline \multirow{3}{*}{ HEIGHT $_{\mathrm{cm}}$} & $\begin{array}{c}\mathrm{X} \\
(\mathrm{SD})\end{array}$ & $\begin{array}{l}170.2 \\
(5.5)\end{array}$ & $\begin{array}{l}170.3 \\
(5.5)\end{array}$ & $\begin{array}{l}170.7 \\
(6.0)\end{array}$ & $\begin{array}{l}168.7 \\
(6.5)\end{array}$ & $\begin{array}{l}168.5 \\
(7.2)\end{array}$ & $\begin{array}{l}168.1 \\
(7.0)\end{array}$ & $\begin{array}{l}169.1 \\
(6.6)\end{array}$ & $\begin{array}{l}167.3 \\
(8.1)\end{array}$ & $\begin{array}{l}169.9 \\
(6.2)\end{array}$ & $\begin{array}{l}168.6 \\
(6.8)\end{array}$ \\
\hline & $\mathrm{AD} \pm \mathrm{SD}$ & \multirow{2}{*}{\multicolumn{2}{|c|}{$\begin{array}{c}-0.1 \pm 0.9 \\
0.978\end{array}$}} & & \multicolumn{2}{|c|}{$0.3 \pm 1.3$} & \multicolumn{2}{|c|}{$1.8 \pm 1.5$} & \multicolumn{2}{|c|}{$1.3 \pm 0.6$} \\
\hline & $\mathrm{p}$ & & & \multicolumn{2}{|c|}{ 0.031* } & \multicolumn{2}{|c|}{0,788} & \multicolumn{2}{|c|}{0.243} & \multicolumn{2}{|c|}{$0.021^{*}$} \\
\hline & $\begin{array}{c}\mathrm{X} \\
(\mathrm{SD})\end{array}$ & $\begin{array}{c}75.3 \\
(11.6)\end{array}$ & $\begin{array}{l}79.7 \\
(9.9)\end{array}$ & $\begin{array}{c}75.8 \\
(11.7)\end{array}$ & $\begin{array}{l}76.3 \\
(8.9)\end{array}$ & $\begin{array}{c}72.0 \\
(10.6)\end{array}$ & $\begin{array}{l}76.2 \\
(8.8)\end{array}$ & $\begin{array}{c}71.4 \\
(11.0)\end{array}$ & $\begin{array}{c}76.9 \\
(11.6)\end{array}$ & $\begin{array}{c}74.3 \\
(11.5)\end{array}$ & $\begin{array}{l}77.2 \\
(9.8)\end{array}$ \\
\hline WEIGHT $_{\mathrm{Kg}}$ & $\mathrm{AD} \pm \mathrm{SD}$ & & & -0.5 & 1.5 & & $=1,8$ & & & -2.9 & 0.9 \\
\hline & $\mathrm{p}$ & & & & & & & & & 0.0 & \\
\hline DMT 2 & $\begin{array}{c}\mathrm{X} \\
(\mathrm{SD})\end{array}$ & $\begin{array}{l}26.0 \\
(3.6)\end{array}$ & $\begin{array}{l}27.3 \\
(3.0)\end{array}$ & $\begin{array}{l}26.0 \\
(3.8)\end{array}$ & $\begin{array}{l}26.8 \\
(3.0)\end{array}$ & $\begin{array}{l}25.3 \\
(3.6)\end{array}$ & $\begin{array}{l}27.0 \\
(3.0)\end{array}$ & $\begin{array}{l}25.1 \\
(3.6)\end{array}$ & $\begin{array}{l}27.5 \\
(3.9)\end{array}$ & $\begin{array}{l}25.7 \\
(3.7)\end{array}$ & $\begin{array}{l}27.1 \\
(3.2)\end{array}$ \\
\hline BMI $\mathrm{Kg} / \mathrm{m}$ & $\mathrm{AD} \pm \mathrm{SD}$ & & & -0.8 & 0.5 & & \pm 0.6 & & & -1.4 & 0.3 \\
\hline & $\mathrm{p}$ & & & & & & & & & 0.0 & $* *$ \\
\hline Resting & $\begin{array}{c}\mathrm{X} \\
(\mathrm{SD})\end{array}$ & $\begin{array}{l}75.8 \\
(7.2)\end{array}$ & $\begin{array}{l}75.3 \\
(7.4)\end{array}$ & $\begin{array}{l}75.7 \\
(7.5)\end{array}$ & $\begin{array}{l}75.2 \\
(7.0)\end{array}$ & $\begin{array}{l}75.7 \\
(8.2)\end{array}$ & $\begin{array}{l}73,9 \\
(8.3)\end{array}$ & $\begin{array}{l}74.3 \\
(8.7)\end{array}$ & $\begin{array}{l}72.6 \\
(9.4)\end{array}$ & $\begin{array}{l}75.5 \\
(7.7)\end{array}$ & $\begin{array}{l}74.3 \\
(8.0)\end{array}$ \\
\hline $\begin{array}{l}\text { Heart } \\
\text { Rate }\end{array}$ & $\mathrm{AD} \pm \mathrm{SD}$ & 0.5 & 1.2 & 0.5 & 1.1 & & 1.3 & & & 1.2 & 0.7 \\
\hline $\mathrm{Kate}_{\mathrm{rate} / \mathrm{min}}$ & $p$ & & & & & & & & & & \\
\hline Effort & $\begin{array}{c}\mathrm{X} \\
(\mathrm{SD})\end{array}$ & $\begin{array}{l}121.7 \\
(12.3)\end{array}$ & $\begin{array}{l}127.1 \\
(13.7)\end{array}$ & $\begin{array}{l}115.8 \\
(10.3)\end{array}$ & $\begin{array}{r}124.6 \\
(10.8)\end{array}$ & $\begin{array}{l}111.8 \\
(8.4)\end{array}$ & $\begin{array}{l}113.8 \\
(12.6)\end{array}$ & $\begin{array}{l}108.9 \\
(9.1)\end{array}$ & $\begin{array}{r}110.9 \\
(11.5)\end{array}$ & $\begin{array}{l}115.6 \\
(11.3)\end{array}$ & $\begin{array}{r}119.3 \\
(13.8)\end{array}$ \\
\hline Heart Rate & $\mathrm{AD} \pm \mathrm{SD}$ & -5.5 & 2.2 & 8.8 & 1.6 & & \pm 1.8 & -2. & 2.2 & -3.7 & 1.1 \\
\hline rate/min & $\mathrm{p}$ & & & 0.0 & & & & & & 0.0 & \\
\hline
\end{tabular}

S: Smokers, NS:Non-Smokers, X: Average, SD: Standard Deviation, AD: Average Difference, p: Significance of differences, ** P $<0.01$ and $* \mathrm{P}<0.05$

Table 3. The Comparison of Test Performances of Smokers and Non-Smokers Based on Their Age Groups

\begin{tabular}{|c|c|c|c|c|c|c|c|c|c|c|c|}
\hline \multirow{2}{*}{\multicolumn{2}{|c|}{$\begin{array}{c}\text { AGE GROUPS } \\
\text { TESTS }\end{array}$}} & \multicolumn{2}{|c|}{$\begin{array}{c}\text { Aged 60-64 } \\
\mathrm{N}=143\end{array}$} & \multicolumn{2}{|c|}{$\begin{array}{c}\text { Aged 65-69 } \\
\mathrm{N}=180 \\
\end{array}$} & \multicolumn{2}{|c|}{$\begin{array}{c}\text { Aged 70-74 } \\
\quad \mathrm{N}=135 \\
\end{array}$} & \multicolumn{2}{|c|}{$\begin{array}{l}\text { Over } 75 \\
N=106\end{array}$} & \multicolumn{2}{|c|}{$\begin{array}{c}\text { Total } \\
\mathbf{N}=564\end{array}$} \\
\hline & & \multirow{2}{*}{$\begin{array}{c}\mathbf{S} \\
\mathrm{n}=69 \\
16.1 \\
(2.9) \\
\end{array}$} & \multirow{2}{*}{$\begin{array}{c}\text { NS } \\
\mathrm{n}=74 \\
16.7 \\
(2.6) \\
\end{array}$} & \multirow{2}{*}{$\begin{array}{c}\begin{array}{c}\mathbf{S} \\
\mathrm{n}=87\end{array} \\
15.0 \\
(2.5)\end{array}$} & \multirow{2}{*}{\begin{tabular}{|c|}
$\mathbf{N S}$ \\
$\mathrm{n}=93$ \\
16.5 \\
$(2.8)$ \\
\end{tabular}} & \multirow{2}{*}{$\begin{array}{c}\mathbf{S} \\
\mathrm{n}=45 \\
13,1 \\
(2.7) \\
\end{array}$} & \multirow{2}{*}{\begin{tabular}{c|c|} 
NS \\
$\mathrm{n}=90$ \\
14.2 \\
$(2.8)$ \\
\end{tabular}} & \multirow{2}{*}{$\begin{array}{c}\begin{array}{c}\mathrm{S} \\
\mathrm{n}=38\end{array} \\
10.9 \\
(3.1)\end{array}$} & \multirow{2}{*}{\begin{tabular}{c|c|} 
NS \\
$\mathrm{n}=68$ \\
12.9 \\
$(3.3)$ \\
\end{tabular}} & \multirow{2}{*}{$\begin{array}{c}\mathbf{S} \\
\mathrm{n}=239 \\
14.3 \\
(3.3)\end{array}$} & \multirow{2}{*}{$\begin{array}{c}\begin{array}{c}\text { NS } \\
\mathrm{n}=325\end{array} \\
15.2 \\
(3.3) \\
\end{array}$} \\
\hline 30 CST & $\begin{array}{c}\mathrm{X} \\
\text { (SD) }\end{array}$ & & & & & & & & & & \\
\hline $\begin{array}{l}\text { Chair Stand } \\
\text { Test }\end{array}$ & $\begin{array}{c}\mathrm{AD} \pm \mathrm{SD} \\
\% \mathrm{AD}\end{array}$ & \multicolumn{2}{|c|}{$\begin{array}{c}-0.6 \pm 0.5 \\
\% 3.6\end{array}$} & \multicolumn{2}{|c|}{$\begin{array}{c}-1.5 \pm 0.4 \\
\% 9.1 \\
\end{array}$} & \multicolumn{2}{|c|}{$\begin{array}{c}-1.1 \pm 0.5 \\
\% 7.8 \\
\end{array}$} & \multicolumn{2}{|c|}{$\begin{array}{c}-2.0 \pm 0.7 \\
\% 15.5 \\
\end{array}$} & \multirow{2}{*}{\multicolumn{2}{|c|}{$\begin{array}{c}-0.8 \pm 0.3 \\
\% 5.9 \\
\end{array}$}} \\
\hline & $\mathrm{P}$ & \multicolumn{2}{|c|}{0.249} & \multicolumn{2}{|c|}{$\mathbf{0 . 0 0 0}^{* *}$} & \multicolumn{2}{|c|}{$0.028^{*}$} & \multicolumn{2}{|c|}{$0.004^{* *}$} & & $0 \mathrm{D.003}^{* *}$ \\
\hline \multirow{3}{*}{$\begin{array}{c}\text { ARCLT } \\
\text { Arm Curl } \\
\text { Test } \\
\text { (number/30s) }\end{array}$} & $\begin{array}{c}\mathrm{X} \\
\mathrm{SD} \\
\end{array}$ & $\begin{array}{l}17.6 \\
(3.1)\end{array}$ & $\begin{array}{l}18.1 \\
(2.3) \\
\end{array}$ & $\begin{array}{l}17.0 \\
(2.3)\end{array}$ & $\begin{array}{l}17.8 \\
(2.5) \\
\end{array}$ & $\begin{array}{l}14.6 \\
(2.6)\end{array}$ & $\begin{array}{l}15.5 \\
(3.0) \\
\end{array}$ & $\begin{array}{l}12.7 \\
(2.1)\end{array}$ & $\begin{array}{l}14.8 \\
(3.3) \\
\end{array}$ & $\begin{array}{l}16.0 \\
(3.1)\end{array}$ & $\begin{array}{l}16.6 \\
(3.1) \\
\end{array}$ \\
\hline & $\begin{array}{c}\mathrm{AD} \pm \mathrm{SD} \\
\% \mathrm{AD}\end{array}$ & \multicolumn{2}{|c|}{$\begin{array}{c}-0.6 \pm 0.5 \\
\% 3.3\end{array}$} & \multicolumn{2}{|c|}{$\begin{array}{c}-0.8 \pm 0.4 \\
\% 4.5\end{array}$} & \multicolumn{2}{|c|}{$\begin{array}{c}-0.9 \pm 0.5 \\
\% 5.8 \\
\end{array}$} & \multicolumn{2}{|c|}{$\begin{array}{c}-2.1 \pm 0.5 \\
\% 14.2 \\
\end{array}$} & \multicolumn{2}{|c|}{$\begin{array}{c}-0.6 \pm 0.3 \\
\% 3.6\end{array}$} \\
\hline & $\mathrm{P}$ & \multicolumn{2}{|c|}{0.210} & & & & & & & & \\
\hline 2MST & $\begin{array}{c}\mathrm{X} \\
\mathrm{SD}\end{array}$ & $\begin{array}{c}70.5 \\
(17.5)\end{array}$ & $\begin{array}{c}83.1 \\
(14.3)\end{array}$ & $\begin{array}{c}64.6 \\
(14.4)\end{array}$ & $\begin{array}{c}80.1 \\
(13.5)\end{array}$ & $\begin{array}{c}56.0 \\
(12.5)\end{array}$ & $\begin{array}{c}66.6 \\
(13.0)\end{array}$ & $\begin{array}{c}43.5 \\
(11.6)\end{array}$ & $\begin{array}{c}55.5 \\
(18.1)\end{array}$ & $\begin{array}{c}61.3 \\
(17.2)\end{array}$ & $\begin{array}{c}71.9 \\
(18.0)\end{array}$ \\
\hline 2-Minute & $\begin{array}{c}\mathrm{AD} \pm \mathrm{SD} \\
\% \mathrm{AD}\end{array}$ & & $\begin{array}{l}2.7 \\
.2\end{array}$ & $\begin{array}{r}-15 . \\
\%\end{array}$ & & $\begin{array}{r}-10 . \\
\%\end{array}$ & & $\begin{array}{r}-12 . \\
\% \\
\end{array}$ & $\begin{array}{l}2.9 \\
.6\end{array}$ & $\begin{array}{r}-10 \\
\%\end{array}$ & \\
\hline & $\mathrm{P}$ & & & & & & & & & & \\
\hline CSRT & $\begin{array}{c}X \\
\text { SD }\end{array}$ & $\begin{array}{l}-3.9 \\
(6.8)\end{array}$ & $\begin{array}{c}0.9 \\
(5.5)\end{array}$ & $\begin{array}{l}-5.6 \\
(8.8)\end{array}$ & $\begin{array}{l}-0.6 \\
(7.9)\end{array}$ & $\begin{array}{l}-9.3 \\
(9.3)\end{array}$ & $\begin{array}{l}-4.5 \\
(8.4)\end{array}$ & $\begin{array}{l}-10.5 \\
(9.0)\end{array}$ & $\begin{array}{l}-5.5 \\
(8.9)\end{array}$ & $\begin{array}{l}-6.7 \\
(8.7)\end{array}$ & $\begin{array}{l}-2.4 \\
(8.2)\end{array}$ \\
\hline $\begin{array}{l}\text { Chair } \\
\text { Sit-and-Reac }\end{array}$ & $\begin{array}{c}\mathrm{AD} \pm \mathrm{SD} \\
\% \mathrm{AD}\end{array}$ & & & & & & & & & & \\
\hline & $\mathrm{P}$ & & & & & & & & & & \\
\hline $\begin{array}{c}\text { BST } \\
\text { SThetch }\end{array}$ & $\begin{array}{c}X \\
\text { SD }\end{array}$ & $\begin{array}{l}-9.6 \\
(8.8)\end{array}$ & $\begin{array}{l}-6.7 \\
(8.7)\end{array}$ & $\begin{array}{c}-9.9 \\
(10.6)\end{array}$ & $\begin{array}{l}-5.5 \\
(8.0)\end{array}$ & $\begin{array}{l}-9.2 \\
(7.9)\end{array}$ & $\begin{array}{l}-10.0 \\
(9.9)\end{array}$ & $\begin{array}{l}-13.3 \\
(10.4)\end{array}$ & $\begin{array}{l}-12.4 \\
(10.2)\end{array}$ & $\begin{array}{l}-10.2 \\
(9.6)\end{array}$ & $\begin{array}{l}-8.5 \\
(9.5)\end{array}$ \\
\hline $\begin{array}{l}\text { Test } \\
(\mathbf{c m})\end{array}$ & $\begin{array}{c}\mathrm{AD} \pm \mathrm{SD} \\
\% \mathrm{AD}\end{array}$ & & & & & & & $\begin{array}{r}-0.9 \\
0 \\
\end{array}$ & & & \\
\hline & $\mathrm{P}$ & & & & & & & & & & \\
\hline 8FUAGT & $\begin{array}{c}\mathrm{X} \\
\mathrm{SD}\end{array}$ & $\begin{array}{c}4.9 \\
(0.8)\end{array}$ & $\begin{array}{l}4.6 \\
(0.7)\end{array}$ & $\begin{array}{c}5.4 \\
(1.1)\end{array}$ & $\begin{array}{l}4.8 \\
(0.7)\end{array}$ & $\begin{array}{c}6.3 \\
(1.4)\end{array}$ & $\begin{array}{c}5.9 \\
(1.2)\end{array}$ & $\begin{array}{c}7.3 \\
(1.4)\end{array}$ & $\begin{array}{c}6.8 \\
(1.9)\end{array}$ & $\begin{array}{c}5.7 \\
(1.4)\end{array}$ & $\begin{array}{c}5.5 \\
(1.5)\end{array}$ \\
\hline $\begin{array}{l}\text { and Go Test } \\
\text { (seconds) }\end{array}$ & $\begin{array}{c}\mathrm{AD} \pm \mathrm{SD} \\
\% \mathrm{AD}\end{array}$ & & & & & & & & & & \\
\hline & $\mathrm{P}$ & & & & & & & & & & \\
\hline
\end{tabular}

S: Smokers, NS: Non-Smokers, X: Average, SD: Standard Deviation, AD: Average Difference, \% OF: Average Difference Percentage, p: Significance of Differences, $* * \mathrm{p}<0.01$, and $* \mathrm{p}<0.05$ 
Table 4. The Comparison of Some Physical and Physiological Features of Smokers and Smoking Quitters Based on Their Age Groups

\begin{tabular}{|c|c|c|c|c|c|c|c|c|c|c|c|}
\hline \multirow{2}{*}{\multicolumn{2}{|c|}{$\begin{array}{c}\text { AGE GROUPS } \\
\begin{array}{c}\text { PARAMETERS } \\
\%\end{array}\end{array}$}} & \multicolumn{2}{|c|}{$\begin{array}{c}\text { Aged 60-64 } \\
\mathrm{N}=129\end{array}$} & \multicolumn{2}{|c|}{$\begin{array}{c}\text { Aged 65-69 } \\
N=185\end{array}$} & \multicolumn{2}{|c|}{$\begin{array}{c}\text { Aged 70-74 } \\
N=120\end{array}$} & \multicolumn{2}{|c|}{$\begin{array}{c}\text { Over } 75 \\
N=90\end{array}$} & \multicolumn{2}{|c|}{$\begin{array}{c}\text { Total } \\
\mathbf{N}=\mathbf{5 2 4}\end{array}$} \\
\hline & & \multirow{2}{*}{$\begin{array}{c}\begin{array}{c}\mathbf{S} \\
\mathrm{n}=69 \\
\% 53.5\end{array} \\
61.6 \\
(1.4)\end{array}$} & \multirow{2}{*}{$\begin{array}{c}\begin{array}{c}\text { SQ } \\
\mathrm{n}=60 \\
\% 46.5 \\
61.7 \\
(1.4)\end{array} \\
\end{array}$} & \multirow{2}{*}{$\begin{array}{c}\begin{array}{c}\mathbf{S} \\
\mathrm{n}=87 \\
\% 47.0\end{array} \\
66.5 \\
(1.4)\end{array}$} & \multirow{2}{*}{ 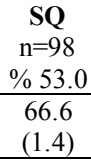 } & \multirow{2}{*}{$\begin{array}{c}\begin{array}{c}\mathbf{S} \\
\mathrm{n}=45 \\
\% 337.5\end{array} \\
71.6 \\
(1.3)\end{array}$} & \multirow{2}{*}{$\begin{array}{c}\begin{array}{c}\text { SQ } \\
\mathrm{n}=75 \\
\% 62.5 \\
72.0 \\
(1.4)\end{array} \\
\end{array}$} & \multirow{2}{*}{$\begin{array}{c}\begin{array}{c}\mathbf{S} \\
\mathrm{n}=38 \\
\% 42.2 \\
77.2 \\
(2.9)\end{array} \\
\end{array}$} & \multirow{2}{*}{\begin{tabular}{|c|}
$\begin{array}{c}\text { SQ } \\
\mathrm{n}=52 \\
\% 557.8\end{array}$ \\
79.2 \\
$(4.0)$
\end{tabular}} & \multirow{2}{*}{$\begin{array}{c}\begin{array}{c}\mathbf{S} \\
\mathrm{n}=239 \\
\% 45.6\end{array} \\
67.7 \\
(5.6)\end{array}$} & \multirow{2}{*}{ 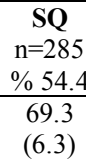 } \\
\hline \multirow{3}{*}{ Age Years } & $\begin{array}{c}\mathrm{X} \\
(\mathrm{SD})\end{array}$ & & & & & & & & & & \\
\hline & $\mathrm{AD} \pm \mathrm{SD}$ & \multirow{2}{*}{\multicolumn{2}{|c|}{$\begin{array}{c}-0.1 \pm 0.2 \\
0.685\end{array}$}} & & & & & -2.0 & & & \\
\hline & $\mathrm{P}$ & & & \multicolumn{2}{|c|}{$\frac{-0.2 \pm 0.2}{0.410}$} & \multicolumn{2}{|c|}{0.104} & \multicolumn{2}{|c|}{ 0.011* } & \multicolumn{2}{|c|}{$0.003 * *$} \\
\hline \multirow{3}{*}{ HEIGHT $_{\mathrm{cm}}$} & $\begin{array}{c}\mathrm{X} \\
\text { (SD) }\end{array}$ & $\begin{array}{l}170.2 \\
(5.5)\end{array}$ & $\begin{array}{c}170.8 \\
(5.9)\end{array}$ & $\begin{array}{l}170.7 \\
(6.0)\end{array}$ & $\begin{array}{c}170.6 \\
(6.2)\end{array}$ & $\begin{array}{l}168.5 \\
(7.2)\end{array}$ & $\begin{array}{c}169.1 \\
(6.7)\end{array}$ & $\begin{array}{l}169.1 \\
(6.6)\end{array}$ & $\begin{array}{c}166.9 \\
(5.6)\end{array}$ & $\begin{array}{l}169.9 \\
(6.2)\end{array}$ & $\begin{array}{l}169.6 \\
(6.3)\end{array}$ \\
\hline & $\mathrm{AD} \pm \mathrm{SD}$ & \multirow{2}{*}{\multicolumn{2}{|c|}{$\begin{array}{c}-0.5 \pm 1.0 \\
0.608\end{array}$}} & \multicolumn{2}{|c|}{$0.1 \pm 0.9$} & \multirow{2}{*}{\multicolumn{2}{|c|}{$\begin{array}{c}-0.7 \pm 1.3 \\
0,614\end{array}$}} & \multirow{2}{*}{\multicolumn{2}{|c|}{$\begin{array}{c}2.2 \pm 1.3 \\
0.091\end{array}$}} & \multirow{2}{*}{\multicolumn{2}{|c|}{$0.3 \pm 0.5$}} \\
\hline & $\mathrm{P}$ & & & & & & & & & & \\
\hline \multirow{3}{*}{ WEIGHT $_{\mathrm{Kg}}$} & $\begin{array}{c}\mathrm{X} \\
\text { (SD) }\end{array}$ & $\begin{array}{c}75.3 \\
(11.6)\end{array}$ & $\begin{array}{r}82.6 \\
(9.8)\end{array}$ & $\begin{array}{c}75.8 \\
(11.7)\end{array}$ & $\begin{array}{c}79.9 \\
(12.0)\end{array}$ & $\begin{array}{c}72.0 \\
(10.6)\end{array}$ & $\begin{array}{c}75.1 \\
(10.3)\end{array}$ & $\begin{array}{c}71.4 \\
(11.0)\end{array}$ & $\begin{array}{c}71.9 \\
(11.4)\end{array}$ & $\begin{array}{c}74.3 \\
(11.5)\end{array}$ & $\begin{array}{c}77.7 \\
(11.6) \\
\end{array}$ \\
\hline & $\mathrm{AD} \pm \mathrm{SD}$ & \multirow{2}{*}{\multicolumn{2}{|c|}{$\begin{array}{c}-7.4 \pm 1.9 \\
\mathbf{0 . 0 0 0 * *}\end{array}$}} & \multirow{2}{*}{\multicolumn{2}{|c|}{$\begin{array}{c}-4.0 \pm 1.8 \\
\mathbf{0 . 0 2 2} *\end{array}$}} & \multirow{2}{*}{\multicolumn{2}{|c|}{$\begin{array}{c}-3.1 \pm 2.0 \\
0.117\end{array}$}} & \multirow{2}{*}{\multicolumn{2}{|c|}{$\begin{array}{c}-0.5 \pm 2.4 \\
0849\end{array}$}} & -3.5 & 1.0 \\
\hline & $\mathrm{p}$ & & & & & & & & & 0.0 & \\
\hline & $\begin{array}{c}\mathrm{X} \\
(\mathrm{SD})\end{array}$ & $\begin{array}{l}26.0 \\
(3.6)\end{array}$ & $\begin{array}{l}28.4 \\
(2.9) \\
\end{array}$ & $\begin{array}{l}26.0 \\
(3.8)\end{array}$ & $\begin{array}{r}27.4 \\
(3.4)\end{array}$ & $\begin{array}{l}25.3 \\
(3.6)\end{array}$ & $\begin{array}{r}26.3 \\
(3.6)\end{array}$ & $\begin{array}{l}25.1 \\
(3.6)\end{array}$ & $\begin{array}{l}25.8 \\
(3.7)\end{array}$ & $\begin{array}{l}25.7 \\
(3.7) \\
\end{array}$ & $\begin{array}{l}27.0 \\
(3.5)\end{array}$ \\
\hline BMI $\mathrm{Kg} / \mathrm{m}$ & $\mathrm{AD} \pm \mathrm{SD}$ & -2. & 0.6 & -1.3 & 0.5 & -1.0 & & -0.7 & & -1.3 & 0.3 \\
\hline & $\mathrm{P}$ & 0.0 & & & & & & 0. & & 0.0 & \\
\hline Resting & $\begin{array}{c}\mathrm{X} \\
(\mathrm{SD})\end{array}$ & $\begin{array}{l}75.8 \\
(7.2)\end{array}$ & $\begin{array}{l}77.4 \\
(8.6)\end{array}$ & $\begin{array}{l}75.7 \\
(7.5)\end{array}$ & $\begin{array}{l}74.5 \\
(7.6)\end{array}$ & $\begin{array}{l}75.7 \\
(8.2)\end{array}$ & $\begin{array}{l}74.7 \\
(8.1)\end{array}$ & $\begin{array}{l}74.3 \\
(8.7)\end{array}$ & $\begin{array}{l}73.2 \\
(9.7)\end{array}$ & $\begin{array}{l}75.5 \\
(7.7)\end{array}$ & $\begin{array}{l}74.9 \\
(8.4)\end{array}$ \\
\hline Heart & $\mathrm{AD} \pm \mathrm{SD}$ & -1.6 & 1.4 & 1.2 & & & & 1.1 & & 0.6 & 0.7 \\
\hline Rate rate/min & $\mathrm{P}$ & & & & & & & 0. & & & \\
\hline Effort & $\begin{array}{c}\mathrm{X} \\
\text { (SD) }\end{array}$ & $\begin{array}{l}121.7 \\
(12.3)\end{array}$ & $\begin{array}{c}124.6 \\
(14.8)\end{array}$ & $\begin{array}{l}115.8 \\
(10.3)\end{array}$ & $\begin{array}{c}116.2 \\
(12.8)\end{array}$ & $\begin{array}{l}111.8 \\
(8.4)\end{array}$ & $\begin{array}{c}114.3 \\
(10.6)\end{array}$ & $\begin{array}{l}108.9 \\
(9.1)\end{array}$ & $\begin{array}{c}109.7 \\
(12.0)\end{array}$ & $\begin{array}{l}115.6 \\
(11.3)\end{array}$ & $\begin{array}{c}116.3 \\
(13.4)\end{array}$ \\
\hline & $\mathrm{AD} \pm \mathrm{SD}$ & -2.9 & 2.4 & -0.4 & 1.7 & -2.5 & & -0.8 & 2.2 & -0.6 & 1.1 \\
\hline & $\mathrm{p}$ & & & & & & & 0 , & & & \\
\hline
\end{tabular}

S: Smokers, SQ: Smoking Quitters, X: Average, SD: Standard Deviation, AD: Average Difference, p: Significance of differences, ${ }^{* *} \mathrm{P}<0.01$ and $*$ $\mathrm{P}<0.05$

Table 5. The Comparison of Test Performances of Smokers and Smoking Quitters Based on Their Age Groups

\begin{tabular}{|c|c|c|c|c|c|c|c|c|c|c|c|}
\hline \multirow{2}{*}{\multicolumn{2}{|c|}{$\begin{array}{c}\text { AGE GROUPS } \\
\text { TESTS }\end{array}$}} & \multicolumn{2}{|c|}{$\begin{array}{c}\text { Aged 60-64 } \\
\text { N= 129 }\end{array}$} & \multicolumn{2}{|c|}{$\begin{array}{c}\text { Aged 65-69 } \\
\text { N= 185 }\end{array}$} & \multicolumn{2}{|c|}{$\begin{array}{c}\text { Aged 70-74 } \\
N=120\end{array}$} & \multicolumn{2}{|c|}{$\begin{array}{c}\text { Over } 75 \\
\mathrm{~N}=90\end{array}$} & \multicolumn{2}{|c|}{$\begin{array}{c}\text { Total } \\
\mathbf{N}=524\end{array}$} \\
\hline & & \multirow{2}{*}{$\begin{array}{c}\mathbf{S} \\
\mathrm{n}=69 \\
16.1 \\
(2.9)\end{array}$} & \multirow{2}{*}{$\begin{array}{c}\text { SQ } \\
\mathrm{n}=60 \\
15.4 \\
(3.1) \\
\end{array}$} & \multirow{2}{*}{$\begin{array}{c}\begin{array}{c}\mathbf{S} \\
\mathrm{n}=87\end{array} \\
15.0 \\
(2.5)\end{array}$} & \multirow{2}{*}{\begin{tabular}{c|c|}
$\mathbf{S Q}$ \\
$\mathrm{n}=98$ \\
15.1 \\
$(3.1)$ \\
\end{tabular}} & \multirow{2}{*}{$\begin{array}{c}\mathbf{S} \\
\mathrm{n}=45 \\
13,1 \\
(2.7) \\
\end{array}$} & \multirow{2}{*}{$\begin{array}{c}\text { SQ } \\
\mathrm{n}=75\end{array}$} & \multirow{2}{*}{ 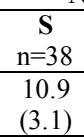 } & \multirow{2}{*}{$\begin{array}{c}\begin{array}{c}\mathbf{S Q} \\
\mathrm{n}=52\end{array} \\
11.8 \\
(2.6)\end{array}$} & \multirow{2}{*}{$\begin{array}{c}\mathbf{S} \\
\mathrm{n}=239 \\
14.3 \\
(3.3)\end{array}$} & \multirow{2}{*}{$\begin{array}{c}\begin{array}{c}\text { SQ } \\
\mathrm{n}=285\end{array} \\
14.1 \\
(3.2) \\
\end{array}$} \\
\hline \multirow{3}{*}{$\begin{array}{c}30 \mathrm{CST} \\
\text { Chair Stand } \\
\text { Test } \\
\text { (number/30s) }\end{array}$} & $\begin{array}{c}\mathrm{X} \\
(\mathrm{SD})\end{array}$ & & & & & & & & & & \\
\hline & $\begin{array}{c}\mathrm{AD} \pm \mathrm{SD} \\
\% \mathrm{AD}\end{array}$ & \multicolumn{2}{|c|}{$\begin{array}{c}0.7 \pm 0.5 \\
\% 4.3\end{array}$} & \multicolumn{2}{|c|}{$\begin{array}{c}-0.1 \pm 0.4 \\
\% 0.7\end{array}$} & \multicolumn{2}{|c|}{$\begin{array}{c}-0.4 \pm 0.5 \\
\% 3.0\end{array}$} & \multicolumn{2}{|c|}{$\begin{array}{c}-0.9 \pm 0.6 \\
\% 7.6\end{array}$} & \multicolumn{2}{|c|}{$\begin{array}{c}0.2 \pm 0.3 \\
\% 1.4 \\
\end{array}$} \\
\hline & $\mathrm{p}$ & \multicolumn{2}{|c|}{0.184} & \multicolumn{2}{|c|}{0.762} & \multicolumn{2}{|c|}{0.499} & \multicolumn{2}{|c|}{0.156} & \multicolumn{2}{|c|}{0.520} \\
\hline \multirow{3}{*}{$\begin{array}{c}\text { ARCLT } \\
\text { Arm Curl } \\
\text { Test } \\
\text { (number/30s) }\end{array}$} & $\begin{array}{c}\mathrm{X} \\
\mathrm{SD}\end{array}$ & $\begin{array}{l}17.6 \\
(3.1)\end{array}$ & $\begin{array}{l}18.3 \\
(2.7)\end{array}$ & $\begin{array}{l}17.0 \\
(2.3)\end{array}$ & $\begin{array}{l}17.0 \\
(2.3)\end{array}$ & $\begin{array}{l}14.6 \\
(2.6)\end{array}$ & $\begin{array}{l}14.7 \\
(2.9)\end{array}$ & $\begin{array}{l}12.7 \\
(2.1)\end{array}$ & $\begin{array}{l}13.9 \\
(2.5)\end{array}$ & $\begin{array}{l}16.0 \\
(3.1)\end{array}$ & $\begin{array}{l}16.1 \\
(3.0)\end{array}$ \\
\hline & $\begin{array}{c}\mathrm{AD} \pm \mathrm{SD} \\
\% \mathrm{AD}\end{array}$ & \multicolumn{2}{|c|}{$\begin{array}{c}-0.7 \pm 0.5 \\
\% 3.8\end{array}$} & \multicolumn{2}{|c|}{$\begin{array}{c}0.0 \pm 0.3 \\
\% 0.0\end{array}$} & \multicolumn{2}{|c|}{$\begin{array}{c}-0.1 \pm 0.5 \\
\% 0.7\end{array}$} & \multicolumn{2}{|c|}{$\begin{array}{c}-1.2 \pm 0.5 \\
\% 8.6\end{array}$} & \multicolumn{2}{|c|}{$\begin{array}{c}-0.1 \pm 0.3 \\
\% 0.6\end{array}$} \\
\hline & $\mathrm{P}$ & & & & & & & & & & \\
\hline 2MST & $\begin{array}{c}\mathrm{X} \\
\mathrm{SD} \\
\end{array}$ & $\begin{array}{c}70.5 \\
(17.5) \\
\end{array}$ & $\begin{array}{c}77.0 \\
(14.3) \\
\end{array}$ & $\begin{array}{c}64.6 \\
(14.4) \\
\end{array}$ & $\begin{array}{r}69.0 \\
(13.4)\end{array}$ & $\begin{array}{c}56.0 \\
(12.5) \\
\end{array}$ & $\begin{array}{c}61.6 \\
(11.8) \\
\end{array}$ & $\begin{array}{c}43.5 \\
(11.6) \\
\end{array}$ & $\begin{array}{c}52.8 \\
(15.3) \\
\end{array}$ & $\begin{array}{c}61.3 \\
(17.2)\end{array}$ & $\begin{array}{c}65.8 \\
(15.7) \\
\end{array}$ \\
\hline $\begin{array}{l}\text { 2-Minute } \\
\text { Step Test }\end{array}$ & $\begin{array}{c}\mathrm{AD} \pm \mathrm{SD} \\
\% \mathrm{AD}\end{array}$ & & 2.8 & & & & & & & $\begin{array}{r}-4.5 \\
\% \\
\end{array}$ & \\
\hline (step/2dak) & $\mathrm{p}$ & & & & & & & & & & \\
\hline CSRT & $\begin{array}{c}\mathrm{X} \\
\mathrm{SD} \\
\end{array}$ & $\begin{array}{l}-3.9 \\
(6.8) \\
\end{array}$ & $\begin{array}{l}-3.3 \\
(8.5)\end{array}$ & $\begin{array}{l}-5.6 \\
(8.8)\end{array}$ & $\begin{array}{l}-5.3 \\
(9.6)\end{array}$ & $\begin{array}{l}-9.3 \\
(9.3)\end{array}$ & $\begin{array}{l}-6.3 \\
(8.9)\end{array}$ & $\begin{array}{l}-10.5 \\
(9.0)\end{array}$ & $\begin{array}{l}-7.7 \\
(9.0)\end{array}$ & $\begin{array}{l}-6.7 \\
(8.7) \\
\end{array}$ & $\begin{array}{l}-5.6 \\
(9.1)\end{array}$ \\
\hline $\begin{array}{c}\text { Chair } \\
\text { Sit-and-Reac }\end{array}$ & $\begin{array}{c}\mathrm{AD} \pm \mathrm{SD} \\
\% \mathrm{AD}\end{array}$ & & & & & & & & & $\begin{array}{r}-1.1 \\
\%\end{array}$ & \\
\hline & $\mathrm{P}$ & & & & & & & & & & \\
\hline BST & $\begin{array}{c}\mathrm{X} \\
\mathrm{SD} \\
\end{array}$ & $\begin{array}{l}-9.6 \\
(8.8)\end{array}$ & $\begin{array}{l}-8.4 \\
(9.4)\end{array}$ & $\begin{array}{c}-9.9 \\
(10.6) \\
\end{array}$ & $\begin{array}{l}-8.4 \\
(9.6)\end{array}$ & $\begin{array}{l}-9.2 \\
(7.9)\end{array}$ & $\begin{array}{l}-12.4 \\
(10.0) \\
\end{array}$ & $\begin{array}{l}-13.3 \\
(10.4) \\
\end{array}$ & $\begin{array}{l}-15.8 \\
(9.0)\end{array}$ & $\begin{array}{l}-10.2 \\
(9.6)\end{array}$ & $\begin{array}{l}-10.8 \\
(9.9) \\
\end{array}$ \\
\hline $\begin{array}{c}\text { Back Scratch } \\
\text { Test }\end{array}$ & $\begin{array}{c}\mathrm{AD} \pm \mathrm{SD} \\
\% \mathrm{AD}\end{array}$ & & & & & & & & & & \\
\hline & $p$ & & & & & & & & & & \\
\hline 8FUAGT & $\begin{array}{c}\mathrm{X} \\
\mathrm{SD}\end{array}$ & $\begin{array}{c}4.9 \\
(0.8)\end{array}$ & $\begin{array}{c}4.7 \\
(0.8)\end{array}$ & $\begin{array}{c}5.4 \\
(1.1)\end{array}$ & $\begin{array}{c}5.2 \\
(0.8)\end{array}$ & $\begin{array}{c}6.3 \\
(1.4)\end{array}$ & $\begin{array}{c}6.0 \\
(1.1)\end{array}$ & $\begin{array}{c}7.3 \\
(1.4)\end{array}$ & $\begin{array}{c}7.2 \\
(2.2)\end{array}$ & $\begin{array}{c}5.7 \\
(1.4)\end{array}$ & $\begin{array}{c}5.7 \\
(1.5)\end{array}$ \\
\hline $\begin{array}{l}\text { 8-Foot Up } \\
\text { and Go }\end{array}$ & $\begin{array}{c}\mathrm{AD} \pm \mathrm{SD} \\
\% \mathrm{AD}\end{array}$ & & & & & & & & & & \\
\hline Test(second) & $\mathrm{P}$ & & & & & & & & & & \\
\hline
\end{tabular}

S: Smokers, SQ: Smoking Quitters, X: Average, SD: Standard Deviation, AD: Average Difference, \% OF: Average Difference Percentage, $p$ : Significance of Differences, $* * \mathrm{p}<0.01$, and $* \mathrm{p}<0.05$ 
Table 6. The Comparison of Some Physical and Physiological Features of Non-Smokers and Smoking Quitters Based on Their Age Groups

\begin{tabular}{|c|c|c|c|c|c|c|c|c|c|c|c|}
\hline \multirow{2}{*}{\multicolumn{2}{|c|}{$\begin{array}{c}\text { AGE GROUPS } \\
\begin{array}{c}\text { PARAMETERS } \\
\% \mathrm{~N}\end{array}\end{array}$}} & \multicolumn{2}{|c|}{$\begin{array}{c}\text { Aged 60-64 } \\
\mathrm{N}=134\end{array}$} & \multicolumn{2}{|c|}{$\begin{array}{c}\text { Aged 65-69 } \\
\quad \mathrm{N}=191\end{array}$} & \multicolumn{2}{|c|}{$\begin{array}{c}\text { Aged 70-74 } \\
\mathrm{N}=165\end{array}$} & \multicolumn{2}{|c|}{$\begin{array}{l}\text { Over } 75 \\
\text { N=120 }\end{array}$} & \multicolumn{2}{|c|}{$\begin{array}{c}\text { Total } \\
\mathbf{N}=610\end{array}$} \\
\hline & & $\begin{array}{c}\mathbf{N S} \\
\mathrm{n}=74 \\
\% 55.2\end{array}$ & $\begin{array}{c}\text { SQ } \\
\mathrm{n}=60 \\
\% 44.8\end{array}$ & $\begin{array}{c}\mathbf{N S} \\
\mathrm{n}=93 \\
\% 487\end{array}$ & $\begin{array}{c}\text { SQ } \\
\mathrm{n}=98 \\
05513\end{array}$ & $\begin{array}{c}\mathbf{N S} \\
\mathrm{n}=90 \\
\% 54.5\end{array}$ & $\begin{array}{c}\text { SQ } \\
\mathrm{n}=75 \\
\% 45.5\end{array}$ & $\begin{array}{c}\mathbf{N S} \\
\mathrm{n}=68 \\
\% 56.7\end{array}$ & $\begin{array}{c}\text { SQ } \\
\mathrm{n}=52 \\
\% 43.3\end{array}$ & $\begin{array}{c}\mathbf{N S} \\
\mathrm{n}=325 \\
\% 53.3\end{array}$ & $\begin{array}{c}\text { SQ } \\
\mathrm{n}=285 \\
\% 467\end{array}$ \\
\hline \multirow{3}{*}{ Age Years } & $\begin{array}{c}\mathrm{X} \\
(\mathrm{SD})\end{array}$ & $\begin{array}{l}61.5 \\
(1.5)\end{array}$ & $\begin{array}{l}61.7 \\
(1.4)\end{array}$ & $\begin{array}{l}66.4 \\
(1.3)\end{array}$ & $\begin{array}{l}66.6 \\
(1.4)\end{array}$ & $\begin{array}{l}71.4 \\
(1.5)\end{array}$ & $\begin{array}{l}72.0 \\
(1.4)\end{array}$ & $\begin{array}{l}78.8 \\
(3.9)\end{array}$ & $\begin{array}{l}79.2 \\
(4.0)\end{array}$ & $\begin{array}{l}69.2 \\
(6.4)\end{array}$ & $\begin{array}{l}69.3 \\
(6.3)\end{array}$ \\
\hline & $\mathrm{AD} \pm \mathrm{SD}$ & \multicolumn{2}{|c|}{$-0.2 \pm 0.3$} & \multicolumn{2}{|c|}{$-0.2 \pm 0.2$} & \multicolumn{2}{|c|}{$-0.7 \pm 0.2$} & \multicolumn{2}{|c|}{$-0.4 \pm 0.7$} & \multicolumn{2}{|c|}{$-0.1 \pm 0.5$} \\
\hline & $\mathrm{P}$ & \multicolumn{2}{|c|}{0.384} & \multicolumn{2}{|c|}{0.220} & \multicolumn{2}{|c|}{$0.004 * *$} & \multicolumn{2}{|c|}{0.592} & \multicolumn{2}{|c|}{0.904} \\
\hline \multirow{3}{*}{ HEIGHT $_{\mathrm{cm}}$} & $\begin{array}{c}\mathrm{X} \\
\text { (SD) }\end{array}$ & $\begin{array}{l}170.3 \\
(5.5)\end{array}$ & $\begin{array}{c}170.8 \\
(5.9)\end{array}$ & $\begin{array}{c}168.7 \\
(6.5)\end{array}$ & $\begin{array}{c}170.6 \\
(6.2)\end{array}$ & $\begin{array}{c}168.1 \\
(6.9)\end{array}$ & $\begin{array}{c}169.1 \\
(6.7)\end{array}$ & $\begin{array}{c}167.3 \\
(8.1)\end{array}$ & $\begin{array}{c}166.9 \\
(5.6)\end{array}$ & $\begin{array}{c}168.6 \\
(6.8)\end{array}$ & $\begin{array}{l}169.6 \\
(6.3)\end{array}$ \\
\hline & $\mathrm{AD} \pm \mathrm{SD}$ & \multicolumn{2}{|c|}{$-0.5 \pm 1.0$} & \multicolumn{2}{|c|}{$-1.9 \pm 0.9$} & \multicolumn{2}{|c|}{$-1.0 \pm 1.1$} & \multicolumn{2}{|c|}{$0.4 \pm 1.3$} & \multicolumn{2}{|c|}{$-1.0 \pm 0.5$} \\
\hline & $\mathrm{P}$ & \multicolumn{2}{|c|}{0.621} & \multicolumn{2}{|c|}{$0.037^{*}$} & \multicolumn{2}{|c|}{0,350} & \multicolumn{2}{|c|}{0.769} & \multicolumn{2}{|c|}{0.068} \\
\hline \multirow{3}{*}{ WEIGHT $_{\mathrm{Kg}}$} & $\begin{array}{c}\mathrm{X} \\
\text { (SD) }\end{array}$ & $\begin{array}{l}79.7 \\
(9.9)\end{array}$ & $\begin{array}{c}82.6 \\
(9.8)\end{array}$ & $\begin{array}{l}76.3 \\
(8.9)\end{array}$ & $\begin{array}{c}79.9 \\
(12.0)\end{array}$ & $\begin{array}{c}76.2 \\
(8.7)\end{array}$ & $\begin{array}{c}75.1 \\
(10.3)\end{array}$ & $\begin{array}{c}76.9 \\
(11.6)\end{array}$ & $\begin{array}{c}71.9 \\
(11.4)\end{array}$ & $\begin{array}{l}77.2 \\
(9.8)\end{array}$ & $\begin{array}{c}77.7 \\
(11.6)\end{array}$ \\
\hline & $\mathrm{AD} \pm \mathrm{SD}$ & \multicolumn{2}{|c|}{$-3.0 \pm 1.7$} & \multicolumn{2}{|c|}{$-3.6 \pm 1.5$} & \multicolumn{2}{|c|}{$1.1 \pm 1.5$} & \multicolumn{2}{|c|}{$5.0 \pm 2.1$} & -0. & 0.9 \\
\hline & $\mathrm{p}$ & & & & & & & & & & \\
\hline 2 & $\begin{array}{c}\mathrm{X} \\
\text { (SD) }\end{array}$ & $\begin{array}{l}27.3 \\
(3.0)\end{array}$ & $\begin{array}{l}28.4 \\
(2.9)\end{array}$ & $\begin{array}{l}26.8 \\
(3.0)\end{array}$ & $\begin{array}{l}27.4 \\
(3.4)\end{array}$ & $\begin{array}{l}27.0 \\
(2.9)\end{array}$ & $\begin{array}{l}26.3 \\
(3.6)\end{array}$ & $\begin{array}{l}27.5 \\
(3.9)\end{array}$ & $\begin{array}{l}25.8 \\
(3.7)\end{array}$ & $\begin{array}{l}27.1 \\
(3.2)\end{array}$ & $\begin{array}{l}27.0 \\
(3.5)\end{array}$ \\
\hline BMII Kg/m & $\mathrm{AD} \pm \mathrm{SD}$ & -1.1 & & -0. & 0.5 & & & & & & 0.3 \\
\hline & $\mathrm{P}$ & & & & & & & & & & \\
\hline Resting & $\begin{array}{c}\mathrm{X} \\
(\mathrm{SD})\end{array}$ & $\begin{array}{l}75.3 \\
(7.4)\end{array}$ & $\begin{array}{l}77.4 \\
(8.6)\end{array}$ & $\begin{array}{l}75.2 \\
(7.0)\end{array}$ & $\begin{array}{l}74.5 \\
(7.6)\end{array}$ & $\begin{array}{l}73.9 \\
(8.3)\end{array}$ & $\begin{array}{l}74.7 \\
(8.1)\end{array}$ & $\begin{array}{l}72.6 \\
(9.4)\end{array}$ & $\begin{array}{l}73.2 \\
(9.7)\end{array}$ & $\begin{array}{l}74.3 \\
(8.0)\end{array}$ & $\begin{array}{l}74.9 \\
(8.4)\end{array}$ \\
\hline Pate & $\mathrm{AD} \pm \mathrm{SD}$ & -2.1 & 1.4 & & 1.1 & & 1.3 & & & -0. & 0.7 \\
\hline Kate $_{\text {rate/min }}$ & $\mathrm{P}$ & & & & & & & & & & \\
\hline Effort & $\begin{array}{c}\mathrm{X} \\
\text { (SD) }\end{array}$ & $\begin{array}{l}127.1 \\
(13.7)\end{array}$ & $\begin{array}{r}124.6 \\
(14.8)\end{array}$ & $\begin{array}{c}124.6 \\
(10.8)\end{array}$ & $\begin{array}{c}116.2 \\
(12.8)\end{array}$ & $\begin{array}{l}113.8 \\
(12.6)\end{array}$ & $\begin{array}{c}114.3 \\
(10.6)\end{array}$ & $\begin{array}{c}110.9 \\
(11.5)\end{array}$ & $\begin{array}{c}109.7 \\
(12.0)\end{array}$ & $\begin{array}{c}119.3 \\
(13.8)\end{array}$ & $\begin{array}{c}116.3 \\
(13.4)\end{array}$ \\
\hline & $\mathrm{AD} \pm \mathrm{SD}$ & 2.5 & & & & -0. & 1.8 & & & & 1.1 \\
\hline rate/min & $\mathrm{P}$ & & & & & & & & & & \\
\hline
\end{tabular}

NS: Non-Smokers, SQ: Smoking Quitters, X: Average, SD: Standard Deviation, AD: Average Difference, p: Significance of differences, ** $\mathrm{P}<0.01$, and* $\mathrm{P}<0.05$

Table 7. The Comparison of Test Performances of Smoker Quitters and Non-Smokers Based on Their Age Groups

\begin{tabular}{|c|c|c|c|c|c|c|c|c|c|c|c|}
\hline \multirow{2}{*}{\multicolumn{2}{|c|}{$\begin{array}{c}\text { AGE GROUPS } \\
\text { TESTS }\end{array}$}} & \multicolumn{2}{|c|}{$\begin{array}{c}\text { Aged 60-64 } \\
N=134\end{array}$} & \multicolumn{2}{|c|}{$\begin{array}{c}\text { Aged 65-69 } \\
\text { N=191 }\end{array}$} & \multicolumn{2}{|c|}{$\begin{array}{l}\text { Aged 70-74 } \\
\mathrm{N}=165\end{array}$} & \multicolumn{2}{|c|}{$\begin{array}{l}\text { Over } 75 \\
N=120\end{array}$} & \multicolumn{2}{|c|}{$\begin{array}{c}\text { Total } \\
\mathbf{N}=610\end{array}$} \\
\hline & & \multirow{2}{*}{$\begin{array}{c}\mathbf{N S} \\
\mathrm{n}=74 \\
16.7 \\
(2.6) \\
\end{array}$} & \multirow{2}{*}{$\begin{array}{c}\text { SQ } \\
\mathrm{n}=60 \\
15.4 \\
(3.1) \\
\end{array}$} & \multirow{2}{*}{$\begin{array}{c}\mathbf{N S} \\
\mathrm{n}=93 \\
16.5 \\
(2.8)\end{array}$} & \multirow{2}{*}{$\begin{array}{c}\begin{array}{c}\text { SQ } \\
\mathrm{n}=98\end{array} \\
15.1 \\
(3.1) \\
\end{array}$} & \multirow{2}{*}{$\begin{array}{c}\mathbf{N S} \\
\mathrm{n}=90 \\
14.2 \\
(2.8)\end{array}$} & \multirow{2}{*}{$\begin{array}{c}\text { SQ } \\
\mathrm{n}=75 \\
13.4 \\
(2.9) \\
\end{array}$} & \multirow{2}{*}{$\begin{array}{c}\mathbf{N S} \\
\mathrm{n}=68 \\
12.9 \\
(3.3)\end{array}$} & \multirow{2}{*}{$\begin{array}{c}\begin{array}{c}\text { SQ } \\
\mathrm{n}=52\end{array} \\
11.8 \\
(2.6)\end{array}$} & \multirow{2}{*}{$\begin{array}{c}\begin{array}{c}\mathbf{N S} \\
\mathrm{n}=325\end{array} \\
15.2 \\
(3.3)\end{array}$} & \multirow{2}{*}{$\begin{array}{c}\begin{array}{c}\text { SQ } \\
\mathrm{n}=285\end{array} \\
14.1 \\
(3.2)\end{array}$} \\
\hline \multirow{3}{*}{$\begin{array}{c}30 \text { CST } \\
\text { Chair Stand } \\
\text { Test } \\
\text { (number/30s) }\end{array}$} & $\begin{array}{c}\mathrm{X} \\
(\mathrm{SD})\end{array}$ & & & & & & & & & & \\
\hline & $\begin{array}{c}\mathrm{AD} \pm \mathrm{SD} \\
\% \mathrm{AD}\end{array}$ & \multicolumn{2}{|c|}{$\begin{array}{c}1.3 \pm 0.5 \\
\% 7.9\end{array}$} & \multicolumn{2}{|c|}{$\begin{array}{c}1.5 \pm 0.4 \\
\% 9.1\end{array}$} & \multicolumn{2}{|c|}{$\begin{array}{c}0.8 \pm 0.4 \\
\% 5.6\end{array}$} & \multicolumn{2}{|c|}{$\begin{array}{c}1.1 \pm 0.6 \\
\% 8.5\end{array}$} & \multicolumn{2}{|c|}{$\begin{array}{c}1.0 \pm 0.3 \\
\% 6.6\end{array}$} \\
\hline & $\mathrm{p}$ & \multicolumn{2}{|c|}{ 0.013* } & \multicolumn{2}{|c|}{$0.001 * *$} & \multicolumn{2}{|c|}{0.092} & \multicolumn{2}{|c|}{0.059} & \multicolumn{2}{|c|}{$0.000 * *$} \\
\hline \multirow{3}{*}{$\begin{array}{c}\text { ARCLT } \\
\text { Arm Curl } \\
\text { Test } \\
\text { (number/30s) }\end{array}$} & $\begin{array}{c}\mathrm{X} \\
\mathrm{SD}\end{array}$ & $\begin{array}{l}18.1 \\
(2.3)\end{array}$ & $\begin{array}{l}18.3 \\
(2.7)\end{array}$ & $\begin{array}{l}17.8 \\
(2.5)\end{array}$ & $\begin{array}{l}17.0 \\
(2.3)\end{array}$ & $\begin{array}{l}15.5 \\
(2.9)\end{array}$ & $\begin{array}{l}14.7 \\
(2.9)\end{array}$ & $\begin{array}{l}14.8 \\
(3.3)\end{array}$ & $\begin{array}{l}13.9 \\
(2.5)\end{array}$ & $\begin{array}{l}16.6 \\
(3.1)\end{array}$ & $\begin{array}{l}16.1 \\
(3.0)\end{array}$ \\
\hline & $\begin{array}{c}\mathrm{AD} \pm \mathrm{SD} \\
\% \mathrm{AD}\end{array}$ & \multicolumn{2}{|c|}{$\begin{array}{c}-0.1 \pm 0.4 \\
\% 0.6\end{array}$} & \multicolumn{2}{|c|}{$\begin{array}{c}0.7 \pm 0.3 \\
\% 3.9\end{array}$} & \multicolumn{2}{|c|}{$\begin{array}{c}0.9 \pm 0.4 \\
\% 5.8\end{array}$} & \multicolumn{2}{|c|}{$\begin{array}{c}0.9 \pm 0.5 \\
\% 6.1\end{array}$} & \multicolumn{2}{|c|}{$\begin{array}{c}0.5 \pm 0.2 \\
\% 3.0\end{array}$} \\
\hline & $\mathrm{P}$ & & & & & & & & & & \\
\hline 2MST & $\begin{array}{c}\mathrm{X} \\
\mathrm{SD}\end{array}$ & $\begin{array}{c}83.1 \\
(14.3) \\
\end{array}$ & $\begin{array}{l}77.0 \\
(14.3)\end{array}$ & $\begin{array}{c}80.1 \\
(13.5)\end{array}$ & $\begin{array}{c}69.0 \\
(13.4)\end{array}$ & $\begin{array}{c}66.6 \\
(13.0)\end{array}$ & $\begin{array}{c}61.6 \\
(11.8) \\
\end{array}$ & $\begin{array}{c}55.5 \\
(18.1)\end{array}$ & $\begin{array}{c}52.8 \\
(15.3)\end{array}$ & $\begin{array}{c}71.9 \\
(18.0)\end{array}$ & $\begin{array}{c}65.8 \\
(15.7)\end{array}$ \\
\hline 2-Minute & $\begin{array}{c}\mathrm{AD} \pm \mathrm{SD} \\
\% \mathrm{AD}\end{array}$ & & & $\begin{array}{r}11 . \\
\%\end{array}$ & & & & & & & \\
\hline & $\mathrm{p}$ & & & & & & & & & 0.0 & \\
\hline CSRT & $\begin{array}{c}\mathrm{X} \\
\mathrm{SD} \\
\end{array}$ & $\begin{array}{c}0.9 \\
(5.5)\end{array}$ & $\begin{array}{l}-3.3 \\
(8.5) \\
\end{array}$ & $\begin{array}{l}-0.6 \\
(7.9)\end{array}$ & $\begin{array}{l}-5.3 \\
(9.6) \\
\end{array}$ & $\begin{array}{l}-4.5 \\
(8.4) \\
\end{array}$ & $\begin{array}{l}-6.3 \\
(8.9) \\
\end{array}$ & $\begin{array}{l}-5.5 \\
(8.9) \\
\end{array}$ & $\begin{array}{l}-7.7 \\
(9.0)\end{array}$ & $\begin{array}{l}-2.4 \\
(8.2)\end{array}$ & $\begin{array}{l}-5.6 \\
(9.1)\end{array}$ \\
\hline $\begin{array}{l}\text { Chair } \\
\text { Sit-and-Reac }\end{array}$ & $\begin{array}{c}\mathrm{AD} \pm \mathrm{SD} \\
\% \mathrm{AD}\end{array}$ & & & & & & & & & & \\
\hline h Test(cm) & $\mathrm{P}$ & & & & & & & & & & \\
\hline BST & $\begin{array}{c}\mathrm{X} \\
\mathrm{SD}\end{array}$ & $\begin{array}{l}-6.7 \\
(8.7)\end{array}$ & $\begin{array}{l}-8.4 \\
(9.4)\end{array}$ & $\begin{array}{l}-5.5 \\
(8.0)\end{array}$ & $\begin{array}{l}-8.4 \\
(9.6)\end{array}$ & $\begin{array}{l}-10.0 \\
(9.9)\end{array}$ & $\begin{array}{l}-12.4 \\
(10.0)\end{array}$ & $\begin{array}{l}-12.4 \\
(10.2)\end{array}$ & $\begin{array}{l}-15.8 \\
(9.0)\end{array}$ & $\begin{array}{l}-8.5 \\
(9.5)\end{array}$ & $\begin{array}{c}-10.8 \\
(9.9)\end{array}$ \\
\hline $\begin{array}{c}\text { Back Scratch } \\
\text { Test }\end{array}$ & $\begin{array}{c}\mathrm{AD} \pm \mathrm{SD} \\
\% \mathrm{AD}\end{array}$ & & & & & & & & & & \\
\hline & $\mathrm{p}$ & & & & & & & & & 0.0 & \\
\hline 8FUAGT & $\begin{array}{c}\mathrm{X} \\
\mathrm{SD} \\
\end{array}$ & $\begin{array}{c}4.6 \\
(0.7)\end{array}$ & $\begin{array}{c}4.7 \\
(0.8) \\
\end{array}$ & $\begin{array}{c}4.8 \\
(0.7)\end{array}$ & $\begin{array}{c}5.2 \\
(0.8) \\
\end{array}$ & $\begin{array}{c}5.9 \\
(1.2)\end{array}$ & $\begin{array}{c}6.0 \\
(1.1) \\
\end{array}$ & $\begin{array}{c}6.8 \\
(1.9)\end{array}$ & $\begin{array}{c}7.2 \\
(2.2) \\
\end{array}$ & $\begin{array}{c}5.5 \\
(1.5) \\
\end{array}$ & $\begin{array}{c}5.7 \\
(1.5)\end{array}$ \\
\hline $\begin{array}{l}\text { 8-Foot Up } \\
\text { and Go Test }\end{array}$ & $\begin{array}{c}\mathrm{AD} \pm \mathrm{SD} \\
\% \mathrm{AD}\end{array}$ & $\begin{array}{r}-0 . \\
0\end{array}$ & $\begin{array}{l}0.1 \\
.1\end{array}$ & $\begin{array}{r}-0 . \\
0\end{array}$ & & & & $\begin{array}{r}-0 . \\
0\end{array}$ & & $\begin{array}{r}-0.2 \\
\%\end{array}$ & \\
\hline & $\mathrm{p}$ & & & & & & & & & & \\
\hline
\end{tabular}

SQ: Smoking Quitters, NS: Non-Smokers, X: Average, SD: Standard Deviation, AD: Average Difference, \% OF: Average Difference Percentage, p: Significance of Differences, ${ }^{* *} \mathrm{p}<0.01$, and ${ }^{*} \mathrm{p}<0.05$ 
When smokers and non-smokers are compared in this study, SFT results demonstrated that non-smokers' performance was statistically higher compared to smokers $(\mathrm{p}<0.01)$ (Table 3). Similarly, when smokers and smoking quitters are compared, it can be clearly observed that smoking quitters displayed better test performance compared to smokers $(p<0.05)$ (Table 5). Finally, when the test performances of non-smokers and smoking quitters are compared, it is evident that better performance values were obtained from non-smokers $(\mathrm{p}<0.05)$ (Table 7). Table 2, 4 and 6 clearly suggest that some of the physical and physiological values of smokers are relatively lower compared to non-smokers and smoking quitters.

\section{Discussion and Conclusions}

As a solution to numerous physical, physiological and mental health problems, it is of vital importance for individuals leading a sedentary lifestyle to do regular exercise and, as a result, exercise is used as a preventive treatment against diseases for all age groups [2].

Ageing can be associated with both cardiorespiratory and musculoskeletal health problems, which are also related to the decline of physical activities [11]. It has been observed that exercise programs consisting of strengthening, balance and flexibility activities positively influenced physical and cognitive functioning in elderly adults [11]. Sedentary time in elderly people is closely related to impaired glucose tolerance, dyslipidemia, high risk waist circumference, coronary heart diseases and low perceived health [11]. Relatively little is known about fitness criteria, particularly on cardiovascular fitness [11]. A strong correlation was found between physical activities in elderly adults (over 50) and cardiovascular diseases, lower risk of diabetes, hypertension and cancer, prevention of falls and higher independency [12]. However, despite the benefits of physical activity during this period of life, physical activities are low [12]. For the most elderly adults (over 75) in the UK, only 1 out of 10 males and 2 out of 20 females meet the criteria for physical activities. Previous studies focusing on the physical activities and sedentary time during lifetime for middle-aged and elderly adults reported that the number of physical activities decreased in parallel with ageing, while sedentary time relatively increased [12]. Factors related to higher physical activity levels in elderly adults include male sex, younger age, marriage, a higher level of education, income and socio-economic status (SES), better physical health, body mass index (BMI) and waist circumference, and lower levels of chronic diseases and depression [12]. Moreover, in a recent systematic review, it was indicated that exercise and leisure time physical activity increased following the retirement [12]. Another cross-sectional study on 1566 males aged 71 and 91 in the UK reported that male smokers who were depressed, were obese or suffered more chronic health conditions spent more sedentary time during a day [12]. Currently, it is not known whether the identified correlations are associated with a sustained physical activity or sedentary behavior, as people age through later life [12]. For the long-term success of interventions, it is essential to understand how these correlations influence sustained activity levels during ageing [12]. The English Longitudinal Study of Ageing (ELSA) offers a unique opportunity to examine patterns in physical activity behaviors in the data were collected using the same instrument every 2 years over a 10 -year period [12].

A study reports that increasing physical activity remarkably reduces mortality by all causes and helps quitting smoking. Increasing physical activity in elderly males is central to both quitting smoking and surviving [13]. Neither smoking nor pack years are predictors of declared physical activity or changes in physical activity after intervention. The results suggest that physical exercise can be recommended as a tool to quit smoking, including elderly males with a long smoking history [9]. Modifiable risk factors such as sedentary lifestyle and smoking carry a serious risk of infection for elderly females, despite the fact that the correlation between them is yet to be proved [10]. While past and current smoking habits are directly linked to abdominal fat in elderly males, it is not observed in elderly females [14]. It was observed that non-smoking males and females, compared to smokers, had 1.6 to 3.6 years and 1.6 to 3.9 years longer life spans, respectively, depending on their level of physical activities. When smokers are excluded, most of non-smokers are not handicapped before their death [15]. A moderate to high level of physical activity are significantly related to longer life spans in smoking males and females $(9.5,10.5$ and 12.9 years for males and 11.1, 12.6 and 15.3 years for females over 65) and in non-smokers (11.0, 14.4 and 16.2 years for males and 12.7, 16.2 and 18.4 years for females over 65 ). A higher level of physical activity is associated with a lower risk of disability before death. These findings clearly demonstrate that quitting smoking and regular physical activity help leading a longer and better life [16]. It was concluded that a long history of smoking may contribute to increased basal plasma noradrenaline (NA) concentrations and a possible rise in sympathetic neural activity in healthy elderly individuals. However, the effect of smoking on plasma NA response induced by exercise is significantly low. The variance of basal plasma NA concentrations among individuals can be partly explained by differences in blood volumes [16]. A study indicates that smokers can walk relatively shorter distances compared to non-smokers. Elderly males with a long history of smoking are recommended to quit smoking by doing regular exercise [9]. Smoking is reported to be positively linked to the abdominal fat in young and middle-aged individuals although such correlation is yet to be established for elderly individuals. Behavioral effect on body fat distribution is crucial due to increasing abdominal fat during ageing and 
related health risks. While past and current smoking habits are directly linked to abdominal fat in elderly males, it is not observed in elderly females [14]. Recent studies demonstrated that increased plasma norepinephrine and sympathetic activity levels in healthy elderly individuals largely resulted from a long history of smoking [17].

In both sexes, a negative correlation was found between bone mineral density values and number of cigarettes smoked per day. A moderate level of physical activity was found to be related to a high bone mineral content in males. Males and females who were physically active in their life had higher bone mineral density compared to those leading a sedentary lifestyle [18]. Ageing and smoking are closely linked to decreased arterial oxygen pressure in horizontal recumbent position and increased oxygen pressure in resting arterio venous oxygen difference. However, at a maximal level of exercise, arterial oxygen pressure and oxygen pressure in arterio venous difference were not heavily affected by ageing and smoking. On the contrary, as an individual became older, dead space and total respiration increased during exercise [19].

Compared to non-smoking males and females, smoking males and females had significantly lower HDL cholesterol levels $(\mathrm{p}<0.01$ ), while heavy smokers had lower HDL cholesterol levels compared to light smokers. The use of multiple linear regression analysis in order to adjust values among age, obesity, alcohol consumption and regular exercise increased the differences between smokers and non-smokers in terms of HDL cholesterol levels. The average values adjusted for males who smoked 20 or more cigarettes per day are lower by $5.3 \mathrm{mg} / \mathrm{dl}(11 \%)$ compared to non-smokers $(\mathrm{p}<0.01)$. Females receiving gonadal hormones were analyzed separately from those who did not receive. In both groups, HDL cholesterol levels of females receiving hormones who smoked 20 or more cigarettes per day were lower by $9.4 \mathrm{mg} / \mathrm{dl}(14 \%)$, while they were lower by $8.6 \mathrm{mg} / \mathrm{dl}(14 \%)$ in non-smoking females (p $<0,01$ for both), which suggests that smoking considerably reduces HDL cholesterol levels. In addition, this correlation is related to the dose received and overlaps other studies, thus demonstrating that a possible causal relation exists between smoking and lower HDL cholesterol level [20].

Three leading factors accelerating ageing are 1) smoking tobacco (particularly cigarettes), 2) lack of exercise and 3) obesity. Furthermore, avoiding smoking and obesity and doing regular exercise not only slows down ageing process but also increases life quality [21]. Current smokers with a body mass index lower than $18.5 \mathrm{~kg} / \mathrm{m}^{2}$ and $35 \mathrm{~kg} / \mathrm{m}^{2}$ runs the risk of a 6 to 8 -fold higher mortality rate compared to non-smokers with a normal body mass index. Smokers with a wide waist circumference have a 5-fold higher mortality risk compared to non-smokers with a waist circumference twice as wider as smokers. Although both smoking and obesity are independent predictors of mortality, the combination of a higher body mass index or
$35 \mathrm{~kg} / \mathrm{m}^{2}$ and a wider waist circumference in current smokers or newly quitters is particularly linked to higher mortality rates [22]. Both male and female constant smokers have lower cardiopulmonary fitness, which is positively linked to the physical activity. Cardiopulmonary fitness in males and females declined at a non-linear rate and started to accelerate after the age of 45 . A higher cardiopulmonary fitness is also associated with a relatively low body mass index, a higher level of physical activity and non-smoking throughout adult lifespan [23, 24]. Body adaptation to physical effort involves a number of functional changes: respiratory, circulatory, metabolic, etc. which should be correlated with the lifestyle and health of the subjects $[25,26,27]$.

When Table 7 is analyzed, it can be observed that SFT values of non- smokers are higher compared to smoking quitters. This finding suggests that smoking may have prolonged effects on an individual's health even after she/he quits it [28]. In sedentary subjects, the long-term effects of smoking might be similar to the effects of physical training [29]. Current smokers engage in fewer exercise sessions per week, exercise for shorter time periods, and, overall, expend fewer kilocalories per week in exercise activities than do former smokers or those who have never smoked [30]. The marginally significant difference in percent body fat, with former smokers having the highest average and current smokers the lowest average percent body fat, is consistent with previous research indicating that smokers tend to be thinner than nonsmokers [31, 32].

In conclusion, as the existing literature also indicates, smoking elderly males had lower physical fitness levels compared to non-smokers and smoking quitters. Therefore, elderly males are strongly recommended to increase their physical activities and quit smoking for a healthier and better life.

\section{REFERENCES}

[1] Eler, N., Eler, S. The Effect of Play Education on Some Physical Features and Growth in Children. Int J SciCult Sport, 5(3):225-232, 2017.

[2] Cavanagh, P., EVANS, J., Fiatarone, M., Hagberg, J., McAuley, E., \& Startzell, J. Exercise and physical activity for elderly adults. Med. Sci. Sports Exerc, 30(6), 1-29, 1998.

[3] Barry, H. C., \& Eathorne, S. W. Exercise and ageing: issues for the practitioner. Medical Clinics of North America, 78(2), 357-376, 1994.

[4] Dionne, I. J., Ades, P. A., \& Poehlman, E. T. Impact of cardiovascular fitness and physical activity level on health outcomes in elderly persons. Mechanisms of ageing and Development, 124(3), 259-267, 2003.

[5] Mcdermott, A. Y., \& Mernitz, H. Exercise and elderly 
patients: prescribing guidelines. American family physician, 74(3):437-444, 2006.

[6] Skelton, D. A. Effects of physical activity on postural stability. Age and ageing, 30(suppl_4), 33-39, 2001.

[7] Spirduso, W. W., \& Cronin, D. L. Exercise dose-response effects on quality of life and independent living in elderly adults. Medicine and Science in Sports and Exercise, 33(6): 598-608, 2001.

[8] Rikli, R. E.; Jones, C. J. Senior fitness test. Champaign (IL): Human Kinetics, 2001

[9] Cooper, T. V., Resor, M. R., Stoever, C. J., \&Dubbert, P. M. Physical activity and physical activity adherence in the elderly based on smoking status. Addictive behaviors, 32(10), 2268-2273, 2007.

[10] Leveille, S. G., Gray, S., LaCroix, A. Z., Ferrucci, L., Black, D. J., \& Guralnik, J. M. Physical inactivity and smoking increase risk for serious infections in elder women. Journal of the American Geriatrics Society, 48(12), 1582-1588, 2000 .

[11] Dogra, S., Clarke, J. M., \& Copeland, J. L. Prolonged sedentary time and physical fitness among Canadian men and women aged 60 to 69. Health Reports, 28(2):3-9, 2017.

[12] Smith, L., Gardner, B., Fisher, A., \& Hamer, M. Patterns and correlates of physical activity behaviour over 10 years in elderlyer adults: prospective analyses from the English Longitudinal Study of Ageing. BMJ open, 5(4): e007423, 2015 .

[13] Holme, I., \& Anderssen, S. A. Increases in physical activity is as important as smoking cessation for reduction in total mortality in elderly men: 12 years of follow-up of the Oslo II study. Br J Sports Med, 49(11):743-748, 2015.

[14] Visser, M., Launer, L. J., Deurenberg, P., \& Deeg, D. J. Past and current smoking in relation to body fat distribution in elder men and women. Journals of Gerontology Series A: Biomedical Sciences and Medical Sciences, 54(6): M293-M298, 1999.

[15] Ferrucci, L., Izmirlian, G., Leveille, S., Phillips, C. L., Corti, M. C., Brock, D. B., \& Guralnik, J. M. Smoking, physical activity, and active life expectancy. American journal of epidemiology, 149(7):645-653, 1999.

[16] Jensen, E. W., Espersen, K., KANSTRUP, L. L., \& Christensen, N. J. Plasma noradrenaline and ageing: effects of smoking habits. European journal of clinical investigation, 26(10): 839-846, 1996.

[17] Christensen, N. J., \& Jensen, E. W. Sympatho adrenal activity and psychosocial stress. Annals of the New York Academy of Sciences, 771(1):640-647, 1995.

[18] Cheng, S., Suominen, H., \& Heikkinen, E. Bone mineral density in relation to anthropometric properties, physical activity and smoking in 75-year-elderly men and women. Ageing Clinical and Experimental Research, 5(1):55-62, 1993.

[19] Malmberg, P., Hedenström, H., \& Fridriksson, H. V. Reference values for gas exchange during exercise in healthy nonsmoking and smoking men. Bulletin European de physiopathologie respiratoire, 23(2):131-138, 1987.

[20] Criqui, M. H., Wallace, R. B., Heiss, G., Mishkel, M., Schonfeld, G., \& Jones, G. T. Cigarette smoking and plasma high-density lipoprotein cholesterol. The Lipid Research Clinics Program Prevalence Study. Circulation, 62(4 Pt 2):IV70-6, 1980.

[21] Ochsner A. "Ageing", J Am Geriatr Soc. 24(9):385-93, 1976.

[22] Koster, A., Leitzmann, M. F., Schatzkin, A., Adams, K. F., van Eijk, J. T., Hollenbeck, A. R., \& Harris, T. B. The combined relations of adiposity and smoking on mortality. The American journal of clinical nutrition, 88(5):1206-1212, 2008.

[23] Jackson, A. S., Sui, X., Hébert, J. R., Church, T. S., \& Blair, S. N. Role of lifestyle and ageing on the longitudinal change in cardiorespiratory fitness. Archives of Internal Medicine, 169(19):1781-1787, 2009.

[24] Eler, N., Zorba, E., Savaș, S. Comparison of the Motoric Features of 10-12 Years Age Female Volleyball Players with Their Technical Capacities. J Ath Per and Nut, 1(1):21-31, 2014

[25] Dusa, F.S., Badau,A., Badau, D., Trambitas, C., Brinzaniuc, K. Investigating the Deformation Parameters of PVC Fitness Balls in Relation to the Height and Body Mass Index of the Users. Mater. Plast, 54(4), 606-609, 2017.

[26] Badau D., Badau A., Paraschıv F., Rogozea L., Al Hussein 1 H., Brinzanuc K. Influences of Vitamin A and $E$ Supplementation on Haematological, Functional and Oxidative Balance Parameters on Handball Players. Rev.Chım. (Bucharest), 69(2):332-336, 2018

[27] Gorucu A., Tokay B., Badau A. The effects of three different types of exercises on aerobic and anaerobic power. Physical education of students, 21(4):152-157, 2017.

[28] Liu Y., Pleasants RA., Croft JB., Wheaton AG., Heidari K., Malarcher AM., Ohar JA., Kraft M., Mannino DM., Strange C. Smoking duration, respiratory symptoms, and COPD in adults aged $\geq 45$ years with a smoking history. International Journal of COPD, 10:1409-1416, 2015.

[29] Gyntelberg F., Meyer J., Relationship Between Blood Pressure and Physical Fitness, Smoking and Alcohol Consumption in Copenhagen Males Aged 40-59. Acta Med Scand, 195:375-380, 1974.

[30] Conway T. L., Cronan T A., Smoking, exercise, and physical fitness. Preventive medicine, 21(6), 723-734, 1992.

[31] Albanes, D., Jones, D. Y., Micozzi, M. S., and M. E. Mattson. Associations between smoking and body weight in the US population: Analysis of NHANES II. American Journal of Public Health, 77:439-444, 1987.

[32] Shimokata, H., Muller, D. C., and Andres, R. (1989). Studies in the distribution of body fat: III. Effects of cigarette smoking. Journal of the American Medical Association, 261:1169-1173, 1989. 